\title{
Multi-Ensayos
}

Vol. 4, $\mathrm{N}^{\circ} 7$

ISSN: 2412-3285

https://multiensayos.unan.edu.ni

DOI: https://doi.org/10.5377/multiensayos.v4i7.9483

\section{La Educación Infantil en Nicaragua, antes y después de los años 80}

\section{Early Childhood Education in Nicaragua, Before and After the 80s}

\author{
Aura Hilda Rayo Pérez ${ }^{1}$
}

Recibido: 2 de marzo de 2018, Aceptado: 27 septiembre de 2018

\section{RESUMEN}

En Nicaragua antes de los años ochenta, la educación era privada, habían muy pocos centros de atención a la niñez, no se contaba con especialistas para atender la primera infancia, generalmente se atendía en casas de habitación donde había una maestra que tenía la vocación, el amor y el interés de enseñar. A estos hogares asistían solo los que tenían posibilidades económicas, porque tenían que pagar. Después del triunfo de la Revolución Popular Sandinista (RPS), se crea el departamento de Educación Preescolar en el Ministerio de Educación Central (ManaguaNicaragua), el gobierno central asigna recursos económicos para profesionalizar a maestros/as, y construir centros para atender a la niñez, tanto en áreas urbanas como rurales. Esto como parte de un proyecto revolucionario que se ha venido fortaleciendo y concientizando en las familias y comunidad en general con la participación activa de las comunidades mismas, y el trabajo realizado por las instituciones y organismos encargados de la educación. El interés fundamental en los diferentes tiempos y actores, ha sido estimular el desarrollo integral que prepare al individuo para la vida.

Palabras claves: niñez; educación infantil; desarrollo integral.

\begin{abstract}
In Nicaragua before the eighties, early childhood education centers were private and there were no specialists in the area. These centers functioned in private houses under the directions of an educator with vocation, love and interest to teach. Children in these centers came from families which could afford the tuition. After the triumph of the Sandinista Popular Revolution (RPS), the Ministry of Education (Managua-Nicaragua) created the Department of Preschool Education. The government allocated economic resources to professionalize teachers, and build centers for preschoolers, both in urban and rural areas. This project has strengthened and raised awareness among individuals allowing the integration of different social actors such as families, local communities, institutions in charge of education. Throughout this time, the fundamental interest of the different actors has been to stimulate the integral development of children to prepare individual for life.
\end{abstract}

Keywords: childhood; early childhood education; integral development.

1 Docente del Departamento de Ciencias de la Educación y Humanidades, UNAN-Mangua/FAREM-Estelí. Correo electrónico: arayo_24@ yahoo.es

Copyright (c) 2018 Revista Multi-Ensayos. 


\section{INTRODUCCIÓN}

Con el presente ensayo se pretende dar a conocer como se ha venido trabajando a través de la historia la Educación Infantil, con la finalidad de reconocer la importancia de una educación adecuada, así mismo, que se valoren las capacidades de aprendizajes y la importancia del estímulo para el desarrollo integral de los individuos desde sus primeros años de vida.

\section{DESARROLLO}

A continuación, una breve descripción de aportes de los tres más grandes filósofos que se interesaron por la atención a la niñez, considerando la relevancia de las capacidades de aprendizajes. Visto desde tres grandes áreas: alma, cuerpo y mente. (Peces, 1986)

Sócrates, uno de los más grandes de la filosofía occidental y universal, maestro de Platón, fundamenta el conocimiento en la experiencia, plantea que la educación, la genética y los hábitos son factores que influyen en la formación durante el desarrollo personal.

Platón, filósofo griego, seguidor de la filosofía de Sócrates y maestro de Aristóteles, fundó una academia en la que participó activamente en el quehacer educativo, su pasión era el arte, la pintura, la poesía y el drama, las que tienen una estrecha relación con las actividades que debe realizar el niño, la niña, para el desarrollo de las diferentes áreas como es: desarrollo motor, cognitivo, social y lenguaje.

La educación es un proceso de socialización de los individuos. En los seis primeros años de vida, el niño y la niña posee un potencial para aprender que no se repite en ningún otro momento de la vida. Es por eso que hay que aprovecharlo al máximo, según Platón. La estimulación de aprendizajes en la niñez, busca fomentar la estructuración del pensamiento y de las formas de expresión. Hoy se habla de educación permanente o continúa, esta establece que el proceso educativo, no se limita a la niñez y juventud, sino a que los seres humanos adquieren conocimientos a lo largo de toda la vida.

Relacionando el pensamiento de Platón con la Educación Infantil actual, es relevante mencionar que no se debe obligar a aprender, se debe mostrar el camino, estimular el pensamiento y los intereses de cada niño, niña, para que haya voluntad de una permanente educación.

El proceso educativo, se basa en una serie de valores que producen cambios intelectuales, emocionales y sociales en el individuo. En la atención a la niñez no se trata de trasmitir conocimientos, sino llevar al individuo hacia la verdad y el bien. Ésta debe ser una constante preocupación de la pedagogía, permitiendo el desarrollo integral cuidadosamente, basado en el entorno del niño, garantizando que haya armonía entre cuerpo y mente.

Aristóteles considera que los deberes indiscutibles del legislador es cuidar y vigilar la educación. Lo cual demuestra que la pedagogía de Aristóteles forma parte de la política. Esta ideología se asocia con los intereses de los gobiernos, en donde se han comprometido a garantizar recursos económicos y humanos para que se dé una educación adecuada, que los niños reciban la atención especializada desde que están en 
el vientre de la madre, al nacer deben ser acogidos en un ambiente apropiado, donde reciban alimentación, amor de sus padres y de la familia. Ningún niño debe quedarse fuera del sistema educativo, práctica que debe vivenciarse en todos los países del mundo. Tal vez en unos más que en otros, pero eso dependerá de las políticas gubernamentales como parte de sus políticas internas.

Aristóteles también considera que el desarrollo del cuerpo precede al del alma, así que determina que se ocupe a los niños en ejercicios gimnásticos, de acuerdo al desarrollo de sus fuerzas físicas y que no se le imponga durante los primeros seis años, ningún trabajo intelectual que exija grandes demostraciones de conocimientos, es una etapa de preparación basado en las capacidades propias de cada niño, niña, en donde los cimientos de estas prácticas se basan en los aportes de filósofos, pedagogos, psicólogos, trabajados por los precursores de la Educación Infantil. Éstos han venido trabajando en el transcurso de la historia, en función del perfeccionamiento de la Educación para la niñez, retomando las características propias de cada individuo.

En Nicaragua también surgieron personajes interesados en el trabajo con la niñez, los que ahora llamamos precursores de la Educación Infantil en Nicaragua. Entre ellos se encuentra Josefa Emilia Toledo Murillo, más conocida como Josefa de Aguerri, maestra normalista. Fue ella quien forjó la pedagogía moderna en Nicaragua.

Dada su comprensión sobre la importancia de la educación desde los primeros años de vida, decide crear centros de atención a niños y niñas. Crea en su casa un jardín infantil, este en Diriamba, donde era su domicilio. Trabaja con los dones de Fröebel, crea una propuesta flexible basaba en los intereses de los niños, aprovechando ampliamente los recursos naturales del medio.

Su metodología de trabajo consistía en que ningún niño llevaba nada a la escuela consigo, ni libros, cuadernos, nunca se les imponía tareas que cumplir en casa. Nada de lección, el niño no está obligado a preocuparse hoy de lo que hizo ayer.

Según Josefa Toledo de Aguerri, no se tortura el entendimiento, el niño no lleva más que a sí mismo. Su naturaleza impresionante y la certeza de que la escuela será hoy tan alegre como ayer. No piensa en la clase hasta el momento en que ésta comienza. Nada de resentimiento por un retraso y todos llegan puntualmente.

Expresa doña Josefa de Aguerri, "Este kindergarten mío, hubiera sido calificado por alguna maestra hostil como un desastre disciplinario, alumnos inquietos que trabajan ¿dónde? ¿Cuándo? y como ellos querían, no estaban enfermos como algunos que vi, rígidos, bien sentados, nerviosos, capaz de alarmar al médico. Respondiendo preguntas que repetían hasta la saciedad ¿Qué es esto? ¿Cómo se llama? ¿De qué está hecho?".

A través de la historia se ha reconocido la importancia de la educación para la niñez, valores básicos para fortalecer el desarrollo de hábitos, habilidades y destrezas, estimulando así la intelectualidad basada en las instrucciones, no en la imposición. 
La Educación Infantil antes llamada, kindergarten, jardines infantiles, Educación Preescolar, ha sido en Nicaragua como en el mundo, toda una historia.

Desde el año 1934 a 1979, Nicaragua estuvo bajo el mando de gobiernos neoliberales, en este período no era prioridad la educación en ninguno de los niveles, y mucho menos para los niños en edad preescolar. Por tanto, estudiaba quien tenía dinero, la educación era privada, muy pocos tenían acceso. La enseñanza en los distintos niveles educativos se basaba en la teoría y poco en la práctica, se trabajaba con un enfoque de educación tradicional, una educación memorística y repetitiva. Los estudiantes absorbían datos sin comprenderlos. No se trabajaba en función de la interpretación, el análisis y mucho menos el aprendizaje basado en la experiencia.

El silencio era un valor en el salón de clases, dialogar iba en contra de los aprendizajes, no se realizaban intercambios de conocimientos maestro-alumno, se consideraba que el docente lo sabía todo, no era una autoridad sino un autoritario en el aula de clases. Las formas de evaluación era única y exclusivamente el examen escrito.

No existían centros de Educación Preescolar, donde se diera atención a la niñez, se hacía en hogares, casas en donde había una muchacha que tenía un cierto nivel de educación y la vocación para trabajar con niños, pero lo trabajaba aplicando una metodología tradicional como se menciona anteriormente, el interés de los padres y madres de familia era que su hijo, hija, aprendiera a leer. La Educación Preescolar ahora Educación Infantil, no estaba basada en el desarrollo de hábitos, habilidades y destrezas, sino en la aceptación de lecciones que la maestra les impartía. Sin embargo, a pesar de trabajar con los pocos recursos metodológicos las maestras hacían lo que estaba en sus manos y en sus conocimientos.

Estas estrategias de trabajo no estimulaban en la niñez el conocimiento basado en la observación, investigación, análisis, contextualización y ni se compartían experiencias como una herramienta de aprendizajes.

El gobierno no destinaba presupuesto para la educación, y mucho menos para la Educación Preescolar, no se capacitaba a los docentes, no se les entregaba material didáctico, la Educación para la niñez no estaba institucionalizada.

El 40\% de la población nicaragüense que tenía cierto grado de educación, era gracias a colegios religiosos y privados establecidos en el país. Algunos funcionaban con ayuda de maestros Españoles. (analfabetismo, www.lavozdelsandinismo.com, 2018).

La Educación Preescolar en Nicaragua se amplía como parte del proceso revolucionario, para poner un ejemplo: en 1,979 asistían a un preescolar 7, 865 niños, diez años después la matrícula era de 65,000 estudiantes entre niños y niñas, área urbana y rural. (Bernheim, 1986).

La Educación Infantil es fundamental para preparar al individuo para la vida, desde los primeros años de vida, se debe poner en práctica el trabajo en equipo, la observación, experimentación, el diálogo entre estudiantes y docente, es importante involucrar a padres y madres de familia en el proceso educativo, 
cuando mejor sea la comunicación entre docentes y familia, mejor serán los aprendizajes de los niños y niñas. La familia juega un papel fundamental en la formación de sus hijos, hijas, creando en ellos una mente sana, estimulando las diferentes áreas de desarrollo mediante la cotidianidad.

Los niños desde el momento del nacimiento quieren conocer descubrir, y esto es lo que la familia debe facilitar, mediante un ambiente adecuado donde reine el amor, la ternura y la comunicación. (Sarlé, 1999).

Cuando el niño llega al sistema educativo regular, lleva una serie de aprendizajes adquiridos en el hogar, es por eso la importancia de que esos aprendizajes puedan ser la base para los nuevos que adquirirá en el sistema regular. La familia y la escuela deben ser la base de la formación sólida y segura para crear y fortalecer de manera vinculada, esto creará seguridad y fortalecerá la personalidad y la autoestima.

Con el triunfo de la Revolución Popular Sandinista (RPS), se reconoce, que un pueblo sin educación es un pueblo sin perspectivas de desarrollo, incapaz de hacer valer sus derechos humanos. Derecho a la educación, salud, cultura y a un trabajo digno. En el año 1979, se encuentra un país con un 53\% de analfabetismo, y a esto se suma que un 7\% de la población solo sabe escribir su nombre, para un total de analfabetismo del $60 \%$.

En este sentido la RPS, asume como una de las primeras tareas, erradicar el analfabetismo. Para esto se da la gran Cruzada Nacional de Alfabetización, trabajo que consistió en la participación de los estudiantes de Educación Media. Éstos se trasladaron al campo junto a sus maestros y maestras para enseñar a leer y escribir a niños, jóvenes, adultos y ancianos.

La Junta de Gobierno de Reconstrucción Nacional, liderada por el comandante de la Revolución Daniel Ortega Saavedra, recordó que los cimientos de la Jornada de Alfabetización se dio cuando el comandante Calos Fonseca Amador, en Pancasán, en el año 1967, orientó a los guerrilleros sandinistas a enseñar a leer y escribir a los campesinos, sector más afectado por el analfabetismo.

Como parte del estudio y trabajo basado en la búsqueda de una atención adecuada a la niñez en Nicaragua, se crean metodologías de trabajo que dieran respuesta a una gran cantidad de niños y niñas que no tenían la oportunidad de asistir a un centro en donde se les brindara atención educativa regular.

Surge entonces la atención a la niñez, entendida como la educación desde una perspectiva integral, oportuna y pertinente que abarca desde el nacimiento hasta los seis años de vida. Se estudia, comprueba y se aplican metodologías de trabajo que puedan contribuir al desarrollo integral a través de diversas modalidades o formas de atención, desde aquella que se potencia en la labor educativa de la familia en sus escenarios cotidianos, hasta las modalidades en ambientes educativos regulares.

El estado retoma el compromiso social de atender con programas multidisciplinarios de salud, educación y alimentación, conceptualizando así, la atención integral del niño, niña y la necesidad de atención en todas sus dimensiones: físicas, intelectuales y psicosociales. 
Ante la demanda creciente del servicio de atención a la niñez por parte de la población, surge la modalidad de la Educación Infantil no Formal, como parte del compromiso con las comunidades y pueblo nicaragüense. A este proyecto se suman organismos internacionales que se sensibilizaron con el proyecto de gobierno del pueblo nicaragüense.

Posteriormente surgen los preescolares Comunales, en los departamentos del norte del país (Nicaragua), atendidos voluntariamente por la comunidad, también los preescolares no escolarizados, que son aquellos que se crearon en comunidades rurales y barrios marginales.

El preescolar popular, es otra forma de dar atención a la niñez, en donde hacían falta centros de atención. Todos los antes mencionados eran financiados por organismos no gubernamentales y apoyados por el gobierno y la comunidad, dado que el país no tenía recursos humanos y materiales en esos momentos para atender dicha demanda.

En 1,990 Nicaragua participa en la Conferencia Mundial sobre Educación para todos y todas, celebrada en Jomtien, Tailandia, donde participaron 155 delegados de diferentes estados del mundo, donde se aprobó el compromiso hemisférico, sobre la atención a la primera infancia y lineamientos programáticos, comprometiéndose así, a trabajar con organizaciones de la sociedad internacional y civil para alcanzar la universalización de la Educación Infantil. (Blanco, 2004)

A medida que ha pasado el tiempo se ha dado mayor y mejor atención a la niñez, también se han capacitado recursos humanos por parte del Ministerio de Educación (MINED) y organismos no gubernamentales que se interesan por la educación del niño y la niña, se ha tomado en serio la educación infantil como la etapa con mayor capacidad de aprendizajes, de manera que en la actualidad, se trabaja en función de que todo niño/a esté integrado en el sistema educativo regular.

La educación se basa en la participación y en la práctica. El estudiante ha pasado de objeto a sujeto, de ser pasivo a ser activo. Existen los avances de la tecnología para investigar. Los niños tanto del campo y la ciudad usan un celular, una computadora, realizan trabajo crítico reflexivo, la observación, investigación, medio de aprendizaje basado en conocimientos previos. Se comparte y se hacen conversatorios, donde aprende el docente y también el estudiante, se aplican técnicas de aprendizajes haciendo uso de material del medio, se contextualizan temas, se trabaja la creatividad y se estimula el desarrollo integral de los individuos desde la temprana edad. (Serrano, 2006)

La matrícula para Educación Infantil aumenta cada año y el gobierno construye y reconstruye cada vez más centros, tanto en el campo como en la ciudad. En la actualidad existen una matrícula de 277,616 niños y niñas.

Tanto los centros de atención a la niñez, urbanos y rurales cuentan con material didáctico que es entregado por el MINED, pero también los y las docentes son capacitados para la elaboración de materiales didácticos, haciendo uso de material del medio, lo que significa que trabajar de manera creativa no es un problema en ningún centro educativo de atención a la niñez, en nuestro país y en la actualidad se especializan docentes para atender niños y niñas en las escuelas normales y en las universidades. 
Nicaragua ha alcanzado logros significativos basados en la importancia de atender la Educación Infantil, se cuenta con un centro de atención a la niñez en cada rincón de Nicaragua, todos los niños y niñas tienen acceso a la educación, tanto en áreas urbanas como rurales, existen docentes capacitados para atender a la niñez de 0 a 6 años. Cada centro cuenta con material didáctico adecuado para realizar diferentes actividades para el desarrollo de un proceso educativo que se basa en el "aprender jugando y el aprender haciendo". El estado garantiza una merienda escolar garantizando así, una alimentación sana y nutritiva.

Se da la atención individualizada, se identifican deficiencias y se brinda atención oportuna, sí el docente lo puede resolver lo hace, si requiere de un profesional de la salud se refiere, previa comunicación con los padres, madres de familia, pero el objetivo es que un niño, niña no se queda sin aprender, por falta de atención.

Aun con los avances alcanzados con mucho esfuerzo, existen problemas, los más comunes son psicosociales, (debido a desintegración de las familias, y la emigración), este es un fenómeno que los docentes atienden con calidad, debido a las capacitaciones brindadas por el MINED. Se hace trabajo con las familias poniendo en práctica la labor en equipo, responsabilidades compartidas, trabajo en comunidad y la búsqueda del bien común.

En la actualidad los padres y las madres de familia llevan a sus hijos e hijas a los centros de atención a la niñez desde muy temprana edad, las familias trabajan y prefieren dejar a sus hijos e hijas en un centro educativo, donde recibirán la atención adecuada y no dejarlos en el hogar donde no recibirán estimulación para su desarrollo integral.

Se ha creado una conciencia donde la comunidad está convencida de la importancia del desarrollo de habilidades que se adquieren mediante la socialización y realización de actividades que solo las maestras les orientan. En todos los centros tienen el material adecuado para que la niñez trabaje, existe interiorización por parte de los padres y las madres, de la importancia del juego, éste no es para pasar el tiempo, sino que es una metodología de trabajo especializada para el desarrollo de aprendizajes de manera divertida.

Cuando los niños llegan al primer grado es evidente la responsabilidad y calidad de trabajo, cuando este ha cursado sus niveles de preescolar, es por eso la preocupación de que todo niño esté integrado en el sistema educativo desde la edad preescolar. También es notoria la seguridad, creatividad, habilidades para hacer, analizar y comprender.

Esto es lo relevante de la Educación Infantil, preparar al individuo para la resolución de problemas, no solo en edad escolar sino para toda su vida. Es verdaderamente un trabajo en equipo entre los diferentes autores de la Educación, funcionarios del MINED, familia, docentes y niños. Acompañar a la niñez durante sus primeros años de vida es todo un reto, hay que atender diferentes ritmos de aprendizaje, conductas, deficiencias y personalidades diferentes, puede ser producto de los contextos y ambientes en donde el niño se ha desarrollado, nada complicado solo se debe contar con las herramientas metodológicas necesarias para brindar el acompañamiento oportuno y pertinente.

En este sentido se tendrán niños y niñas felices, adolescentes, jóvenes y adultos preparados para enfrentar las adversidades de la vida. Adultos críticos y autocríticos capaces de resolver problemas, y también 
ser agentes de cambio que contribuyan al desarrollo económico, político y social de un país que se ha preocupado por el desarrollo basado en la educación.

\section{CONCLUSIONES}

La educación infantil en Nicaragua ha alcanzado relevante importancia tanto por parte de las instituciones encargadas de la educación, como por la comunidad educativa. (Padres, madres, sociedad, funcionarios de la educación).

Estos logros se han alcanzado mediante un proceso de concientización en la población sobre la importancia de formar hombre y mujeres desde los primeros años de vida, trabajar la solidaridad, la cooperación, la responsabilidad, disciplina, trabajo en equipo, formar líderes, todo lo antes mencionado es trabajo de los especialistas en Educación Infantil. Para el ser humano es mucho más fácil poner en práctica estos valores cuando se practican desde los primeros años de vida.

Los niños y niñas tienen la mayor capacidad de aprendizajes entre las edades de 0 a 6 años, esta etapa de la vida se debe aprovechar al máximo, en ningún otro momento de sus vidas tendrán las mismas capacidades de aprender.

En este sentido es que se ha trabajado en función de crear las bases en la atención a la niñez, en cada rincón de Nicaragua, hay un centro de atención, y están muy bien equipados con material didáctico, las y los docentes capacitados para hacer uso adecuado del material y trabajar también con los diferentes recursos que se encuentren en los contextos. El material del medio es otro recurso que se ha aprendido a utilizar para el estímulo de los aprendizajes específicos en niños y niñas que llevan al desarrollo de hábitos, habilidades y destrezas, que serán utilizadas para la resolución de problemas en las adversidades de la vida del ser humano.

Para que haya hombres y mujeres profesionales de calidad, hay que brindar una Educación Infantil de calidad.

“Lo que se dé a los niños, los niños darán a la sociedad” Karl A. Meninger

\section{BIBLIOGRAFÍA}

Analfabetismo, N. 1. (28 de Febrero de 2018). www.lavozdelsandinismo.com. Obtenido de www. lavozdelsandinismo.com.

Bernheim, C. T. (1986). Panorama general sobre la Filosofía de la Educación. Managua: Hispamer.

Blanco, R. (20 de Febrero de 2004). Obtenido de http//repositorio.minedu.gob.

Garcia, B.R. (25 de Febrero de 2017). http//repositorio.unan.edu.ni. Obtenido de Repositorio. unan.edu. ni.

Peces, S. L. (1986). Didáctica Magna. España: Akal.

Sarlé, P. M. (1999). Juego y aprenidzaje escolar. Argentina: Novedades Educativas.

Serrano, R. M. (2006). La convergencia europea y la formación universitaria en Competencias para la docencia en Educación Infantil. Educación, 03. 